\title{
THE ENDOMORPHISMS OF THE TOTAL OPERATOR DOMAIN OF AN INFINITE MODULE
}

\author{
BY \\ R. E. JOHNSON AND F. KIOKEMEISTER
}

The total operator domain of a finite vector space of dimensionality $n$ over a division ring is a total matrix algebra of order $n^{2}$ over a division ring anti-isomorphic with the given ring. The only nonzero endomorphisms of this total matrix algebra are the inner automorphisms, the automorphisms induced by automorphisms of the division ring, and products of these two $\left.{ }^{1}\right)$.

If we now start with an infinite vector space with Hamel basis over a division ring $P$, the total operator domain can be thought of as a matrix algebra of infinite order over a division algebra $\bar{P}$ anti-isomorphic with $P$. Since the choice of elements in the infinite matrices is restricted, the matrix algebra should perhaps not be called total, though it is a maximal ring contained in the set of all infinite matrices with elements in $\bar{P}$. The present paper is a study of the endomorphisms of this total operator domain.

To avoid the assumption of the well-ordering of any set, the infinite vector space is assumed to have a countable Hamel basis over $P$. Most of the methods introduced and the results carry over, however, for a basis of any cardinal number if the well-ordering assumption is used. As in the finite case, it is shown that the only nonzero endomorphisms are meromorphisms of the domain. However, the meromorphisms need not be automorphisms.

A formula is given for all meromorphisms of the operator ring. Simplifications occur in the cases of $\bar{P}$-meromorphisms and automorphisms. In the latter case the results are the same as those for the finite matrix algebras.

The total operator domain has no nonzero anti-endomorphisms.

1. Introduction. If $\boldsymbol{\Xi}$ is a module with nonzero submodules $\Xi_{1}, \Xi_{2}, \ldots$ and if every nonzero element $\xi$ of $\Xi$ has a unique form of representation $\xi=\eta_{r_{1}}+\eta_{r_{2}}+\cdots+\eta_{r_{n}}$ as the sum of nonzero elements $\eta_{r_{i}}$ lying in $\Xi_{r_{i}}$, we shall say that $\Xi$ is the direct sum of the submodules $\Xi_{1}, \Xi_{2}, \cdots$ and shall write

$$
\Xi=\Xi_{1}+\Xi_{2}+\cdots=\sum_{i} \Xi_{i}
$$

Let $P$ be a division ring and let $\Xi$ be a regular $P$-module. Then $\alpha \xi=0$ for $\alpha$ in $P$ and $\xi$ in $\Xi$ if and only if $\alpha=0$ or $\xi=0$. Further, the identity $\epsilon$ of $P$ must be an identity operator for $\Xi$, for if $\epsilon \xi \neq \xi$, then $\epsilon(\xi-\epsilon \xi)=0$ while $\epsilon \neq 0$ and $\xi-\epsilon \xi \neq 0$.

Presented to the Society, April 27, 1946; received by the editors February 11, 1946, and, in revised form, November 8, 1946.

(') N. Jacobson, The theory of rings, Mathematical Surveys, vol. 2, New York, 1943, p. 23. 
If $\xi_{1}, \xi_{2}, \ldots$ are elements in $\Xi, P\left(\xi_{1}, \xi_{2}, \cdots\right)$ will designate the least $P$ submodule of $\Xi$ containing $\xi_{1}, \xi_{2}, \cdots$ and will consist of all finite sums $\alpha_{1} \xi_{1}+\alpha_{2} \xi_{2}+\cdots+\alpha_{n} \xi_{n}, \alpha_{i}$ in $P$. For $\xi$ in $\Xi, P \xi$ denotes the $P$-module generated by $\xi$. The elements $\xi_{1}, \xi_{2}, \cdots$ are linearly independent if

$$
P\left(\xi_{1}, \xi_{2}, \cdots\right)=P \xi_{1}+P \xi_{2}+\cdots .
$$

In case there exist elements $\xi_{1}, \xi_{2}, \ldots$ in $\Xi$ such that

$$
\Xi=P \xi_{1}+P \xi_{2}+\cdots
$$

then the set of elements $\xi_{1}, \xi_{2}, \ldots$ is called a proper $P$-basis of $\Xi$. We shall assume that $\Xi$ has a countably infinite proper $P$-basis throughout this paper. Hence if $\xi$ is in $\Xi$ and $\Xi$ has the basis (1.1) then $\xi=\sum_{i=1}^{n} \alpha_{i} \xi_{i}, \alpha_{i}$ in $P$, and if $\sum_{i=1}^{n} \alpha_{i} \xi_{i}=\sum_{i=1}^{n} \beta_{i} \xi_{i}$, then $\alpha_{i}=\beta_{i}, i=1,2, \cdots, n$.

It is known that the $P$-submodules of $\Xi$ will have certain properties $\left({ }^{2}\right)$.

(A) Every $P$-submodule of $Z$ has a countable proper $P$-basis and the number of elements in the basis is an invariant of the submodule.

(B) If $\Xi_{1}$ is a $P$-submodule of $\Xi$, there exists a $P$-submodule $\Xi_{2}$ such that $\Xi=\Xi_{1}+\Xi_{2}$; that is, $\Xi$ is completely reducible with respect to $P$.

A mapping of $\Xi$ into itself or part of itself, $\xi \rightarrow \xi^{\prime}$, is a $P$-endomorphism if and only if, for $\xi \rightarrow \xi^{\prime}, \eta \rightarrow \eta^{\prime}, \alpha$ in $P$,

$$
\begin{gathered}
\xi+\eta \rightarrow \xi^{\prime}+\eta^{\prime}, \\
\alpha \xi \rightarrow \alpha \xi^{\prime} .
\end{gathered}
$$

This endomorphism will be represented by an operator $a ; \xi \rightarrow a \xi$. With this convention (1.2) and (1.3) can be written

$$
\begin{gathered}
a(\xi+\eta)=a \xi+a \eta, \\
\alpha(a \xi)=a(\alpha \xi) .
\end{gathered}
$$

The set of all $P$-endomorphisms of $\Xi$ is called the total operator domain of $\Xi$ and is labelled $\Delta$. Elements of $\Delta$ are denoted by $a, b, c, \cdots$; the elements $a, b$ of $\Delta$ are equal, $a=b$, if and only if $a \xi=b \xi$ for all $\xi$ in $\Xi$. As is well known, $\Delta$ is a ring under the operations of addition and multiplication defined by

$$
\begin{gathered}
(a+b) \xi=a \xi+b \xi, \\
(a b) \xi=a(b \xi) .
\end{gathered}
$$

The unity element of $\Delta$ is denoted by $e$, the zero element by 0 .

Consider now the proper $P$-basis of $\Xi$ given in (1.1). Any element $a$ of $\Delta$ obviously determines a mapping $\xi_{i} \rightarrow a \xi_{i}$ of the basis elements into $\Xi$. A converse of this statement is now given.

(2) M. H. Ingraham, A general theory of linear sets, Trans. Amer. Math. Soc. vol. 27 (1925) p. 178. 
THEOREM 1.1. A mapping of the elements of a proper P-basis of $\Xi$ into $\Xi$ can be extended to a unique $P$-endomorphism of $\mathbf{\Xi}$.

Proof. Let $\xi_{i} \rightarrow m\left(\xi_{i}\right)$ be a mapping of the basis (1.1) into $\Xi$. If $a$ is the mapping of $\Xi$ defined by

$$
a\left(\sum_{i} \alpha_{i} \xi_{i}\right)=\sum_{i} \alpha_{i} m\left(\xi_{i}\right)
$$

then $a$ is a $P$-endomorphism and $a$ is an extension of $m$ as $a \xi_{i}=m\left(\xi_{i}\right)$. Evidently $a$ is unique.

The mapping $\xi \rightarrow \alpha \xi$, $\alpha$ in $P$, of $\Xi$ is a $P$-endomorphism if $P$ is a field. In this case, $P$ can be considered a subring of $\Delta:$ it is easily shown that $P$ is the center of $\Delta$. However, if $P$ is a division ring, the mapping $\xi \rightarrow \alpha \xi, \alpha$ in $P$, of $\Xi$ is not in general a $P$-endomorphism. A situation somewhat analogous to the case of $P$ a field is obtained by defining as in Theorem 1.1 the $P$-endomorphism $\bar{\alpha}$ for the basis (1.1) as

$$
\bar{\alpha} \xi_{i}=\alpha \xi_{i}, \quad i=1,2, \ldots .
$$

The ring $\bar{P}=\{\bar{\alpha} ; \alpha \in P\}$ is a division subring of $\Delta$ and is anti-isomorphic to $P$. It is important to note that $\bar{P}$ is independent on the choice of basis of $\Xi$, that is, $\bar{\alpha} \xi$ and $\alpha \xi$ are in general unequal. The center $C$ of $P$ is a field as is the center $\bar{C}$ of $\bar{P}$. It is apparent that $C$ and $\bar{C}$ are isomorphic. If $\xi$ is any element of $\Xi$,

$$
\xi=\sum_{i=1}^{n} \gamma_{i} \xi_{i}
$$

and if $\bar{\alpha}$ is in $\bar{C}$, then

$$
\alpha \xi=\sum_{i=1}^{n} \gamma_{i} \bar{\alpha} \xi_{i}=\sum_{i=1}^{n} \gamma_{i} \alpha \xi_{i}=\alpha \xi .
$$

We may therefore identify $\bar{C}$ with $C$. This will be done in the future.

2. Preliminary discussion of $Z$ and $\Delta$. If $a$ is in $\Delta$ and $Z_{1}$ is a $P$-submodule of $\boldsymbol{\Xi}$, then the set $a \boldsymbol{\Xi}_{1}$ of all elements of the form $a \xi, \xi$ in $\boldsymbol{\Xi}_{1}$, is a $P$-module.

Definition 2.1. The order of a $P$-submodule $\Xi_{1}$ of $\Xi$ is the number of elements in a proper $P$-basis of $Z_{1}$, and is either a positive integer or $\aleph_{0}$ depending on whether the proper $\boldsymbol{P}$-basis has a finite or infinite number of elements. The order of $\Xi_{1}$ is designated by $o\left(\Xi_{1}\right)$. The order of an element $a$ of $\Delta$ is the order of $a \Xi$, that is, $o(a)=o(a \Xi)$.

Lemma 2.1. If $\Xi_{1}$ and $\Xi_{2}$ are $P$-submodules of $\Xi$ and if $a$ and $b$ are in $\Delta$, then:

(1) $o\left(\Xi_{2}\right) \leqq o\left(\Xi_{1}\right)$ if $\Xi_{2} \subset \Xi_{1}$

(2) $o\left(\Xi_{1}, \Xi_{2}\right) \leqq o\left(\Xi_{1}\right)+o\left(\Xi_{2}\right)$, 
(3) $o\left(\Xi_{1}+\Xi_{2}\right)=o\left(\Xi_{1}\right)+o\left(\Xi_{2}\right)$ if $\Xi_{1} \cap \Xi_{2}=0$,

(4) $o\left(a \Xi_{1}\right) \leqq o\left(\Xi_{1}\right)$,

(5) $o(a b) \leqq o(a)$,

(6) $o(a b) \leqq o(b)$,

(7) $o(a+b) \leqq o(a)+o(b)$.

Proof. (1)-(3) are obvious. If $\eta_{1}, \eta_{2}, \cdots$ is a proper $P$-basis of $\boldsymbol{\Xi}_{1}$, then the set $a \eta_{1}, a \eta_{2}, \cdots$ includes a proper $P$-basis of $a \Xi_{1}$. Hence (4) follows readily. As $(a b) \Xi$ is contained in $a \Xi$, (5) follows from (1). If we replace $\Xi_{1}$ by $b \Xi$ in (4) we obtain (6). As $(a+b)$ is contained in $(a \Xi, b \bar{\Xi})$, (7) follows from (2).

Corresponding to any element $a$ in $\Delta$ there is a set $H_{a}$ contained in $\Xi$ made up of all elements of $\Xi$ that are annihilated by $a$. If $a \xi=a \eta=0$, then $a(\alpha \xi+\beta \eta)=0$ for any $\alpha, \beta$ in $P$. Thus $H_{a}$ is a $P$-module.

LEMMA 2.2. To each element $a$ in $\Delta$ there corresponds a decomposition of $\Xi$ into a direct sum:

$$
\Xi=\sum_{i} P \delta_{i}+H_{a},
$$

where $a \Xi$ has the proper P-basis $a \delta_{1}, a \delta_{2}, \ldots$.

Proof. Since $H_{a}$ is a $P$-submodule of $Z$, there exists a $P$-submodule $Z_{1}$ such that $\boldsymbol{\Xi}=\Xi_{1}+H_{a}$. Then $a \boldsymbol{\Xi}=a \boldsymbol{\Xi}_{1}$. If $\boldsymbol{\Xi}_{1}=\sum_{i} P \delta_{i}$, the elements $a \delta_{1}, a \delta_{2}, \cdots$ must be linearly independent since $a\left(\sum_{i} \alpha_{i} \delta_{i}\right)=0$ if and only if $\sum_{i} \alpha_{i} \delta_{i}$ is in $H_{a}$ which in turn is true if and only if $\alpha_{i}=0$ for all $i$. Hence $a \Xi=\sum_{i} P\left(a \delta_{i}\right)$.

LEMMA 2.3. To each element $a$ in $\Delta$ and to each decomposition (2.1) of $\Xi$ there corresponds on element $\bar{a}$ in $\Delta$ such that:

(1) $a \bar{a}$ and $\bar{d} a$ are idempotent.

(2) $a \bar{d} a=a, \bar{a} a \bar{a}=\bar{a}$.

(3) $o(a \bar{a})=o(\bar{d} a)=o(a)$.

(4) $a d \delta_{i}=\delta_{i}, i=1,2, \cdots$.

Proof. Select the $P$-submodule $\Xi_{1}$ so that $\Xi=a \Xi+\Xi_{1}$. We have, from Lemma 2.2, $\Xi=\sum_{i} P \delta_{i}+H_{a}, a \Xi=\sum_{i} P\left(a \delta_{i}\right)$. Define $\bar{a}$ to be the element of $\Delta$ for which

$$
a\left(a \delta_{i}\right)=\delta_{i}, i=1,2, \cdots ; a \Xi_{1}=0 .
$$

By Theorem 1.1, $\bar{a}$ exists. Immediately we have that

$$
(\bar{d} a) \delta_{i}=\delta_{i}, i=1,2, \cdots ;(\bar{d} a) H_{a}=0,
$$

and $\bar{a} a$ is idempotent. Further

$$
(a \bar{a}) a \delta_{i}=a \delta_{i}, i=1,2, \cdots ;(a \bar{a}) \Xi_{1}=0,
$$

so that $a \bar{a}$ is also idempotent.

Now $o(a)$ is the number of elements in the set $a \delta_{1}, a \delta_{2}, \cdots$ as is $o(a \bar{a})$, 
while $o(\bar{a} a)$ is the number of elements in the set $\delta_{1}, \delta_{2}, \cdots$. Thus $o(a)=o(a \bar{a})$ $=o(\bar{a} a)$. Also

$$
\begin{aligned}
\bar{a}\left(a \delta_{i}\right) & =(\bar{a} a \bar{a})\left(a \delta_{i}\right)=\delta_{i}, i=1,2, \cdots ; \bar{a} \Xi_{1}=(\bar{a} a \bar{a}) \Xi_{1}=0, \\
a \delta_{i} & =(a \bar{a} a) \delta_{i}, i=1,2, \cdots ; \quad a H_{a}=(a \bar{a} a) H_{a}=0,
\end{aligned}
$$

from which we conclude $\bar{a}=\bar{a} a \bar{a}, a=a \bar{a} a$.

Conclusion (2) of this lemma shows that $\Delta$ is a regular ring in the sense of von Neuman $\left({ }^{3}\right)$.

We see in passing that if $a$ is an idempotent element of $\Delta$, then

$$
\Xi=a \Xi+H_{a} \text {. }
$$

This is a consequence of the Peirce decomposition $\xi=a \xi+(\xi-a \xi)$, where $a \xi$ lies in $a \boldsymbol{\Xi}, \xi \neg a \xi$ lies in $\boldsymbol{H}_{\boldsymbol{a}}$.

LEMMA 2.4. If $e_{1}$ and $e_{2}$ are idempotent elements of $\Delta$ for which $o\left(e_{1}\right)=o\left(e_{2}\right)$, then there exist elements $d_{1}$ and $d_{2}$ of $\Delta$ such that:

(1) $o\left(d_{1}\right)=o\left(d_{2}\right)=o\left(e_{1}\right)$,

(2) $d_{1}=d_{1} e_{1}=e_{2} d_{1}, d_{2}=d_{2} e_{2}=e_{1} d_{2}$.

(3) $e_{1}=d_{2} d_{1}, e_{2}=d_{1} d_{2}$.

(4) $e_{1}=d_{2} e_{2} d_{1}, e_{2}=d_{1} e_{1} d_{2}$.

Proof. Let $\Xi=\sum_{i} P \delta_{i}+H_{e_{1}}=\sum_{i} P \gamma_{i}+H_{e_{2}}$ with $e_{1} \delta_{i}=\delta_{i}, \quad e_{2} \gamma_{i}=\gamma_{i}$, $i=1,2, \cdots$. Define $d_{1}$ and $d_{2}$ to be the elements of $\Delta$ for which

$$
\begin{aligned}
& d_{1} \delta_{i}=\gamma_{i}, i=1,2, \cdots ; d_{1} H_{e_{1}}=0, \\
& d_{2 \gamma_{i}}=\delta_{i}, i=1,2, \cdots ; d_{2} H_{e_{2}}=0 .
\end{aligned}
$$

This is possible since $o\left(e_{1}\right)=o\left(e_{2}\right)$. Then (1) and (2) follow immediately. Also

$$
\begin{aligned}
d_{2} d_{1} \delta_{i} & =\delta_{i}, i=1,2, \cdots ; d_{2} d_{1} H_{e_{1}}=0, \\
d_{1} d_{2} \gamma_{i} & =\gamma_{i}, i=1,2, \cdots ; d_{1} d_{2} H_{e_{2}}=0,
\end{aligned}
$$

so that (3) follows. (4) is a consequence of (2) and (3).

In case one of the idempotent elements of this lemma is the unity $e$ of $\Delta$, we have the following corollary.

Corollary. If $a$ is an idempotent element of $\Delta$ with $o(a)=o(e)$, then there exist elements $s$ and $t$ in $\Delta$ such that:

(1) $e=t s, a=s t$.

(2) $s=a s, t=t a$.

(3) $e=$ tas.

LEMMA 25. Let $a$ and $b$ be any two elements of $\Delta$ and suppose $o(a) \leqq o(b)$. Then there exist elements $c_{1}$ and $c_{2}$ in $\Delta$ such that $a=c_{1} b c_{2}$.

(') J. von Neumann, On regular rings, Proc. Nat. Acad. Sci. U. S. A. vol. 22 (1936) p. 707. 
Proof. Select decompositions (2.1) corresponding to $a$ and $b$ : $\Xi$ $=\sum_{i} P \delta_{i}+H_{a}$ with $a \mathbf{\Xi}=\sum_{i} P\left(a \delta_{i}\right) ; \Xi=\sum_{i} P \gamma_{i}+H_{b}$ with $b \Xi=\sum_{i} P\left(b \gamma_{i}\right)$. Define $c$ to be the element of $\Delta$ such that

$$
\begin{array}{rlr}
c \gamma_{i} & =\gamma_{i}, & i=1,2, \cdots, o(a), \\
c \gamma_{i} & =0 & \text { for } i>o(a) \text { in case } o(a)<o(b), \\
c H_{b} & =0 . &
\end{array}
$$

Thus $c$ is idempotent and $o(c)=o(a)$.

By Lemma 2.3 there exists $b$ such that $b b \gamma_{i}=\gamma_{i}, i=1,2, \cdots, b b H_{b}=0$. It follows that $c \bar{b} b=c$. Using the same lemma, an element $\bar{a}$ exists such that $\bar{a} a$ is idempotent, $a=a \bar{a} a$, and $o(\bar{a} a)=o(a)=o(c)$. By Lemma 2.4 there exist elements $d_{1}$ and $d_{2}$ such that $\bar{a} a=d_{1} c d_{2}$. Hence

$$
a=a \bar{a} a=a d_{1} c d_{2}=a d_{1} c b b d_{2},
$$

. and the lemma follows for $c_{1}=a d_{1} c b$ and $c_{2}=d_{2}$.

COROLlaRY. If $a$ is any element of $\Delta$ for which $o(a)=o(e)$ there exist elements $p$ and $q$ in $\Delta$ such that

$$
p a q=e .
$$

To any proper $P$-basis $\xi_{1}, \xi_{2}, \cdots$ of $\Xi$ there corresponds a set of elements $e_{i j}, i, j=1,2, \cdots$, of $\Delta$ defined by

$$
e_{i} \xi_{j}=\xi_{i}, \quad e_{i j} \xi_{k}=0,
$$$$
j \neq k ; i, j, k=1,2, \cdots .
$$

These elements have the well known multiplication table

$$
e_{i j} e_{j k}=e_{i k}, \quad e_{i j} e_{n k}=0, \quad j \neq n ; i, j, k, n=1,2, \cdots,
$$

with the elements $e_{i i}, i=1,2, \cdots$, being idempotent. Obviously $o\left(e_{i j}\right)=1$ for all $i$ and $j$, and $o\left(e_{11}+e_{22}+\cdots+e_{n n}\right)=n$.

Lemma 2.6. The set, $\Omega$, of elements a in $\Delta$ for which $o(a)<o(e)$ is a proper subring of $\Delta$.

Proof. To say that $o(a)<o(e)$ is to say that $o(a)$ is finite. Lemma 2.1 guarantees that this set is a ring. Since it does not contain $e$ and since it contains at least the elements $e_{i j}$ defined above, it is a proper subring of $\Delta$.

THEOREM 2.1. The ring $\Omega$ is the only proper ideal contained in $\Delta$.

Proof. If $a$ is in $\Omega$, then $a b$ and $b a$ are in $\Omega$ for any $b$ in $\Delta$. For, by Lemma $2.1, o(b a) \leqq o(a), o(a b) \leqq o(a)$. Hence $\Omega$ is an ideal.

If $I$ is an ideal of $\Delta$ not contained in $\Omega$, there exists an element $a$ in $I$ such that $o(a)=o(e)$. By the corollary to Lemma 2.5 there exist elements $p$ and $q$ in $\Delta$ such that $p a q=e$ and $I$ contains $e: I=\Delta$. If $I$ is a proper ideal of $\Delta$ contained in $\Omega, I$ contains an element $a$ of positive order $o(a)$. Then $o\left(e_{i i}\right) \leqq o(a)$ 
and, by Lemma $2.5, I$ must contain all $e_{i i}, i=1,2, \ldots$. Hence $I$ contains $e_{11}+e_{22}+\cdots+{ }_{n n}$ for all integers $n$ and as this element is of order $n$ we may argue that $I$ contains all elements of order less than or equal to $n$ for arbitrary $n$, that is, $I=\Omega$.

Definition 2.2. The countable set $a_{1}, a_{2}, \cdots$ of elements of $\Delta$ is said to be algebraically summable if and only if to every $\xi$ in $\Xi$ there corresponds an integer $n=N(\xi)$ such that $a_{n}$ is in the set and $a_{k} \xi=0$ for every element $a_{k}$ of the set for which $k>N(\xi)$. If $a_{1}, a_{2}, \cdots$ is algebraically summable, then $a_{1}+a_{2}+\cdots$ is given by

$$
\left(a_{1}+a_{2}+\cdots\right) \xi=a_{1} \xi+a_{2} \xi+\cdots+a_{n} \xi, \quad n=N(\xi) .
$$

It is evident from this definition that every finite set of elements of $\Delta$ is algebraically summable, and if $a_{1}, a_{2}, \cdots$ is algebraically summable, $a_{1}+a_{2}+\cdots$ is an element of $\Delta$.

Consider now the basis of $\boldsymbol{Z}$ given in (1.1) and the corresponding elements $e_{i j}$ of $\Delta$ given in (2.2) and (2.3). Corresponding to this basis of $Z$ is a division ring $\bar{P}$ contained in $\Delta$ and anti-isomorphic to $P$. Now for $\bar{\alpha}$ in $\bar{P}$,

$$
\begin{array}{r}
\alpha e_{i j} \xi_{j}=\alpha \xi_{i}=\alpha \xi_{i}=e_{i j} \alpha \xi_{i}=e_{i j} \bar{\alpha} \xi_{j}, \quad \alpha e_{i j} \xi_{k}=e_{i j} \bar{\alpha} \xi_{k}=0, \\
i, j, k=1,2, \cdots ; j \neq k,
\end{array}
$$

so that $\bar{\alpha} e_{i j}=e_{i j} \bar{\alpha}$ for all $i, j$. That is, the elements of $\bar{P}$ commute with all $e_{i j}$.

If $a$ is an element of $\Delta$, there exist elements $\alpha_{i j}$ in $P$ such that

$$
a \xi_{j}=\sum_{i=1}^{n i} \alpha_{i j} \xi_{i}, \quad j=1,2, \cdots
$$

Let $a_{j}$ be defined by

$$
a_{j}=\sum_{i=1}^{n i} \bar{\alpha}_{i j} e_{i j}, \quad j=1,2, \cdots
$$

Now $a_{j} \xi_{k}=0$ for $j \neq k$ so that the set $a_{1}, a_{2}, \ldots$ is algebraically summable. Further

$$
a \xi_{j}=\left(a_{1}+a_{2}+\cdots\right) \xi_{j}=a_{j} \xi_{j}=\sum_{i=1}^{n_{j}} \alpha_{i j} \xi_{i}, \quad j=1,2, \cdots,
$$

and it follows that $a=a_{1}+a_{2}+\cdots$. This can be expressed in a double summation as

$$
a=\sum_{j=1}^{\infty} \sum_{i=1}^{n_{j}} \bar{\alpha}_{i j} e_{i j}
$$

We shall frequently abbreviate this to $a=\sum \bar{\alpha}_{i j} e_{i j}$.

TheOREM 2.2. The set of elements $e_{i j}, i, j=1,2, \cdots$, is a $\bar{P}$ basis for $\Delta$ 
under the operation of algebraic summation. Further, if $a$ is given by (2.4), then

$$
e_{r s} a e_{i j}=\bar{\alpha}_{s i} e_{r j}, \quad \quad i, j, r=1,2, \ldots ; s=1,2, \ldots, n_{i} \text {. }
$$

From (2.5) we derive that the only elements of $\Delta$ that commute with all $e_{i j}$ are the elements of $\bar{P}$. For if $a$ is given by (2.4) and $a e_{i j}=e_{i j} a$, $i, j=1,2, \cdots$, then, from (2.5), $\bar{\alpha}_{s i}=0$ if $s \neq i$, and $\bar{\alpha}_{r r} e_{r k}=e_{r k} \bar{\alpha}_{k k k}$. Hence $a=\bar{\alpha}_{r r}, r=1,2, \cdots$, and $a$ is in $\bar{P}$.

THEOREM 2.3. If the sets $a_{1}, a_{2}, \cdots$ and $b_{1}, b_{2}, \cdots$ are algebraically summable and $c, c_{1}, c_{2}, \cdots$ are arbitrary elements of $\Delta$, then the sets $a_{1}+b_{1}$, $a_{2}+b_{2}, \cdots, c_{1} a_{1}, c_{2} a_{2}, \cdots$ and $a_{1} c, a_{2} c, \cdots$ are algebraically summable and

$$
\begin{aligned}
\left(a_{1}+a_{2}+\cdots\right)+\left(b_{1}+b_{2}+\cdots\right) & =\left(a_{1}+b_{1}\right)+\left(a_{2}+b_{2}\right)+\cdots, \\
a_{1} c+a_{2} c+\cdots & =\left(a_{1}+a_{2}+\cdots\right) c \\
c a_{1}+c a_{2}+\cdots & =c\left(a_{1}+a_{2}+\cdots\right) .
\end{aligned}
$$

3. Endomorphisms of $\Delta$. The principal topic of this paper is the discussion of the endomorphisms of $\Delta$. For completeness we define all terms.

Definition 3.1. A mapping $\theta$ of $\Delta$ into a subring $\Delta^{\theta}$ of $\Delta$ is an endomorphism if and only if for $a, b$ in $\Delta$,

$$
\begin{aligned}
(a+b)^{\theta} & =a^{\theta}+b^{\theta}, \\
(a b)^{\theta} & =a^{\theta} b^{\theta} .
\end{aligned}
$$

If in addition

$$
a^{\theta}=b^{\theta}
$$

if and only if $a=b$

then $\theta$ is a meromorphism. An automorphism is a meromorphism $\theta$ for which $\Delta^{\theta}=\Delta$. An endomorphism $\theta$ is complete if and only if whenever the set $a_{1}, a_{2}, \cdots$ is algebraically summable,

$$
\text { the set } a_{1}^{\theta}, a_{2}^{\theta}, \cdots \text { is algebraically summable, }
$$

$$
\left(a_{1}+a_{2}+\cdots\right)^{0}=a_{1}^{0}+a_{2}^{0}+\cdots \cdot
$$

If (ii) be replaced by

$$
(a b)^{\theta}=b^{\theta} a^{\theta}
$$

then $\theta$ is an anti-endomorphism. An endomorphism $\theta$ is a $\bar{P}$-endomorphism (relative to the basis (1.1)) if and only if for $\bar{\alpha}$ in $\bar{P}$,

$$
(\bar{\alpha} a)^{\theta}=\bar{\alpha} a^{\theta},(a \bar{\alpha})^{\theta}=a^{\theta} \bar{\alpha} .
$$

If $u$ and $v$ are elements of $\Delta$ such that $v u=e$, then the correspondence $\theta$ defined by

$$
a^{0}=u c v
$$


is a meromorphism. We shall call $\theta$ an inner meromorphism. If and only if $u v=v u=e$ will $\theta$ be an inner automorphism.

A set of meromorphisms $\theta_{1}, \theta_{2}, \cdots$ of $\Delta$ is said to be orthogonal if and only if

$$
e^{\theta_{i}} e^{\theta_{j}}=0, \quad i \neq j, i, j=1,2, \cdots .
$$

If the set $e^{\theta_{1}}, e^{\theta_{2}}, \cdots$ is algebraically summable, then as $a^{\theta_{i}}=a^{\theta_{i}} e^{\theta_{i}}$ the set $a^{\theta_{1}}, a^{\theta_{2}}, \ldots$ is also algebraically summable as a consequence of Theorem 2.3.

Definition 3.2. If the set of meromorphisms $\theta_{1}, \theta_{2}, \cdots$ is orthogonal and if the set $e^{\theta_{1}}, e^{\theta_{2}}, \cdots$ is algebraically summable, then the correspondence $\theta$ defined by

$$
a^{\theta}=a^{\theta_{1}}+a^{\theta_{2}}+\cdots
$$

is called the direct sum of the meromorphisms $\theta_{1}, \theta_{2}, \cdots$ and is written

$$
\theta=\theta_{1} \oplus \theta_{2} \oplus \cdots \text {. }
$$

THEOREM 3.1. If $\theta$ is the direct sum of the meromorphisms $\theta_{1}, \theta_{2}, \cdots$ then $\theta$ is a meromorphism of $\Delta$. If $\theta_{1}, \theta_{2}, \cdots$ are $\bar{P}$-meromorphisms, then $\theta$ is a $\bar{P}$ meromorphism.

Proof. For any elements $a, b$ in $\Delta$,

$$
a^{\theta_{i} b^{\theta_{j}}}=\left(a^{\theta_{i}} e^{\theta_{i}}\right)\left(e^{\theta_{i} b^{\theta_{j}}}\right)=0, \quad i \neq j, i, j=1,2, \cdots .
$$

Thus

$$
a^{\theta} b^{\theta}=\left(\sum_{i} a^{\theta_{i}}\right)\left(\sum_{i} b^{\theta_{i}}\right)=\sum_{i} a^{\theta_{i} b^{\theta_{i}}}=(a b)^{\theta} .
$$

Also

$$
a^{\theta}+b^{\theta}=\sum_{i} a^{\theta_{i}}+\sum_{j} b^{\theta_{i}}=\sum_{i}(a+b)^{\theta_{i}}=(a+b)^{\theta}
$$

in view of (2.6). If $a^{\theta}=b^{\theta}$, then $e^{\theta_{i} b^{\theta}}=e^{\theta_{i}} a^{\theta}$ so that $a^{\theta_{i}}=b^{\theta_{i}}$. As $\theta_{i}$ is a meromorphism, $a=b$. Hence $\theta$ is a meromorphism of $\Delta$.

The second part of the theorem follows immediately from Theorem 2.3.

It was shown in Theorem 2.2 that the elements $e_{i j}, i, j=1,2, \cdots$, constitute a $\bar{P}$-basis for $\Delta$ under the operation of algebraic summation. The completeness of an endomorphism $\theta$ allows us to select a similar basis for $\Delta^{\theta}$. For if $a$ is any element of $\Delta$ expressed in the form (2.4), then

$$
a^{\theta}=\sum_{j=1}^{\infty} \sum_{i=1}^{n_{j}} \bar{\alpha}_{i j}^{\theta} e_{i j}^{\theta}
$$

Hence we can assert that the elements $e_{i j}^{\theta}, i, j=1,2, \cdots$, form a $\bar{P}^{\theta}$-basis for $\Delta^{\theta}$ under the operation of algebraic summation. 
An endomorphism that is not a meromorphism is said to be proper. A trivial proper endomorphism is $a^{\theta}=0$ for all $a$ in $\Delta$. This is the zero endomorphism. The next few pages will be devoted to the task of showing that the zero endomorphism is the only proper endomorphism of $\Delta$ and also that every endomorphism of $\Delta$ is complete.

If $\theta$ is a proper endomorphism of $\Delta$, there exist elements $b_{1}$ and $b_{2}$ in $\Delta$, $b_{1} \neq b_{2}$, such that $b_{1}^{\theta}=b_{2}^{\theta}$. An equivalent statement is that there exists a nonzero element $b$ in $\Delta$ such that $b^{\theta}=0$, that is, $b=b_{1}-b_{2}$. Express $b$ in the form $b=\sum \bar{\beta}_{i j} e_{i j}$. As $b$ is unequal to zero, there exist integers $r$ and $s$ such that $\bar{\beta}_{r s} \neq 0$. From (2.5)

$$
\left(\bar{\beta}_{r s}\right)^{-1} e_{m r} b e_{s n}=e_{m n}, \quad m, n=1,2, \cdots .
$$

An application of the endomorphism $\theta$ yields $e_{m n}^{\theta}=0$, for all $m, n$.

If in addition to being proper 6 is also complete, then (3.1) shows that $a^{\theta}=0$ for every $a$ in $\Delta$, and we have established the following theorem.

TheOREM 3.2. A nonzero complete endomorphism of $\Delta$ is necessarily a meromorphism.

We temporarily divert our attention from the main topic of the paper to establish a lemma that is needed in the next theorem. Let the set of positive integers $(1,2,3, \cdots)$ be denoted by $\rho$. For any subset $\pi$ of $\rho$, let the vector $I_{\pi}$ be defined by

$$
I_{*}=\left(\epsilon_{1}, \epsilon_{2}, \cdots, \epsilon_{n}, \cdots\right)
$$

where

$$
\epsilon_{n}=\epsilon, \quad n \in \pi ; \quad \epsilon_{n}=0, \quad n \notin \pi,
$$

$\boldsymbol{\epsilon}$ being the identity of $P$.

Define the vector space $Q$ as

$$
Q=P\left[I_{\pi} ; \pi \subset \rho\right]
$$

so that $Q$ is the least vector space over $P$ that contains $I_{\pi}$ for every subset $\pi$ of $\rho$.

Lemma 3.1. The vector space $Q$ does not have a countable P-basis.

Proof. If $Q$ has a countable proper $P$-basis: $Q=P \zeta_{1}+P \zeta_{2}+\cdots$ then define

$$
\zeta_{i}^{\prime}=\zeta_{i}-\sum_{j<i} \gamma_{j i} \zeta_{i}
$$

where the $\gamma_{j i}$ are selected so that $\zeta_{i}^{\prime}$ has the maximum possible number of successive zeros as its first coordinates. The set $\zeta_{1}^{\prime}, \zeta_{2}^{\prime}, \cdots$ must also be a proper $P$-basis for $Q$, as no two of the $\zeta_{i}^{\prime}$ can have the same number of suc- 
cessive zeros as their first coordinates. Now the vectors $\left(\epsilon_{1}, \epsilon_{2}, \cdots, \epsilon_{n}, \cdots\right)$ where $\epsilon_{n}=\epsilon$ and $\epsilon_{k}=0, k \neq n, n=1,2, \cdots$, must be in $Q$. Hence it must be possible to reorder the new basis and possibly multiply by elements of $P$ so that it becomes $\eta_{1}, \eta_{2}, \cdots$, where

$$
\eta_{k}=\left(0,0, \cdots, \epsilon, \alpha_{k+1}, \alpha_{k k+2}, \cdots\right),
$$

$\epsilon$ being the $k$ th coordinate.

To show that $Q=P \eta_{1}+P \eta_{2}+\cdots$ is impossible, let $\mu_{1}=0, \nu_{1}=\epsilon$ and recursively

$$
\mu_{n}=\sum_{i=1}^{n-1} \nu_{i} \alpha_{i n}, \quad\left\{\begin{array}{r}
\nu_{n}=\epsilon \text { if } \mu_{n}=0, \\
\nu_{n}=-\mu_{n} \text { if } \mu_{n} \neq 0 .
\end{array}\right.
$$

Select the vector

$$
I=\left(\epsilon_{1}, \epsilon_{2}, \cdots, \epsilon_{n}, \cdots\right)
$$

so that

$$
\epsilon_{n}=\epsilon \text { if } \mu_{n}=0, \quad \epsilon_{n}=0 \text { if } \mu_{n} \neq 0 .
$$

If, as we are assuming, $I=\sum_{i=1}^{N} \beta_{i} \eta_{i}$, a direct computation of $\beta_{1}, \beta_{2}, \cdots, \beta_{N}$ shows that $\beta_{i}=\nu_{i}$ so that $I=\sum_{i-1}^{N} \nu_{i} \eta_{i}$. However, then

$$
\epsilon_{N+1}=\sum_{i=1}^{N} \nu_{i} \alpha_{i N+1}=\mu_{N+1},
$$

which is not possible as $\epsilon_{k} \neq \mu_{k}$ for all values of $k$. Thus $I$ is not in $P \eta_{1}+P \eta_{2}$ $+\cdots$ and $Q$ cannot have a countable $P$-basis.

Incidentally, we note that $Q$ is not in general the set of all vectors $\left(\alpha_{1}, \alpha_{2}, \cdots, \alpha_{n} \cdots\right)$ over $P$. For example, the vector $(\epsilon, 2 \epsilon, \cdots, n \epsilon, \cdots)$ is not in $Q$ if $P$ is not a finite field. Obviously $Q$ is the set of all countably infinite vectors in case $P$ is a finite field.

Consider now an endomorphism $\theta$ of $\Delta$. We shall establish the fact that $\theta$ must be a complete endomorphism. To this end, let $\pi_{1}, \pi_{2}, \cdots$ be any countable partition of $\rho$, the set of positive integers. That is

$$
\pi_{1} \cup \pi_{2} \cup \ldots=\rho, \quad \pi_{i} \cap \pi_{i} \text { void, } \quad i \neq j, i, j=1,2, \ldots .
$$

Select a proper $P$-basis for $\Xi$, say (1.1), and the corresponding set, $e_{i j}$, of elements of $\Delta$ defined in (2.2). Let

$$
e\left(\pi_{i}\right)=\sum_{i \in \pi_{i}} e_{j i}, \quad i=1,2, \cdots
$$

THEOREM 3.3. The set $e^{\theta}\left(\pi_{1}\right), e^{\theta}\left(\pi_{2}\right), \cdots$ is algebraically summable.

Proof. The theorem is obvious if the partition of $\rho$ is finite, so let us assume an infinite partition, that is, an infinite number of $\pi_{i}$. 
If the set $e^{\theta}\left(\pi_{1}\right), e^{\theta}\left(\pi_{2}\right), \cdots$ were not algebraically summable, there would exist a $\xi_{j}$ of the basis (1.1), say $\xi_{1}$ for convenience, and an infinite set of positive integers, $i_{1}, i_{2}, \cdots$, such that

$$
e^{\theta}\left(\pi_{n}\right) \xi_{1} \neq 0,
$$$$
n=i_{1}, i_{2}, \cdots
$$

By Theorem 2.2, we can write

$$
e^{\theta}\left(\pi_{i_{n}}\right)=\sum_{r, e} \bar{\alpha}_{n r e} e_{r e}
$$$$
n=1,2, \cdots
$$

From (1), for every integer $n$ there exists an integer $k_{n}$ such that

$$
\bar{\alpha}_{n k_{n} 1} \neq 0 \text {, }
$$$$
n=1,2, \cdots \text {. }
$$

Let $\pi$ be any subset of $\rho$, and define

$$
\bar{e}(\boldsymbol{\pi})=\sum_{n \in \pi} e\left(\pi_{i_{n}}\right) .
$$

This is algebraically summable, as, for any integer $k, e\left(\pi_{n}\right) \xi_{k}$ is unequal to zero for exactly one value of $n$. Again $\bar{e}^{\theta}(\pi)$ can be written as

$$
\bar{e}^{\theta}(\pi)=\sum_{r, s} \bar{\beta}_{x r s} e_{r s}
$$

The elements $e\left(\pi_{i}\right), \tilde{e}(\pi)$ are idempotent, and

$$
\begin{aligned}
e^{\theta}\left(\pi_{i_{n}}\right) \vec{e}^{\theta}(\pi) & =e^{\theta}\left(\pi_{i_{n}}\right) & & (n \in \pi) \\
& =0 & & (n \notin \pi) .
\end{aligned}
$$

The substitution of (2) and (3) in (4) yields

$$
\begin{aligned}
\sum_{i} \bar{\alpha}_{n r i} \bar{\beta}_{\pi j e} & =\bar{\alpha}_{n r s} & & (n \in \pi) \\
& =0 & & (n \in \pi),
\end{aligned}
$$

$r, s=1,2, \cdots$. The summation on $j$ is finite for all values of $r, s$ and $\pi$ and is independent of $n$ as we see from (3). In particular, if $r=k_{n}$ and $s=1$, (5) takes on the form

$$
\begin{aligned}
\sum_{j=1}^{t_{n}} \bar{\alpha}_{n k_{n} i \bar{\beta}_{x i 1}} & =\bar{\alpha}_{n k_{n} 1} & & (n \in \pi) \\
& =0 & & (n \notin \pi) .
\end{aligned}
$$

For convenience of notation we let

$$
\begin{array}{lr}
\bar{\alpha}_{n i}=\left(\bar{\alpha}_{n k_{n} 1}\right)^{-1} \bar{\alpha}_{n k, i}, & i=1,2, \cdots, \\
\bar{\beta}_{n i}=\bar{\beta}_{\pi i 1}, & i=1,2, \cdots, t_{n} .
\end{array}
$$

Then $\left(5^{\prime}\right)$ can be written as 


$$
\sum_{j=1}^{i \pi} \bar{\alpha}_{n j} \bar{\beta}_{\pi j}=\epsilon_{n}, \quad \begin{cases}\epsilon_{n}=\epsilon, & n \in \pi, \\ \epsilon_{n}=0, & n \notin \pi .\end{cases}
$$

Let the vectors $\zeta_{i}$ be defined by

$$
\zeta_{i}=\left(\bar{\alpha}_{1 i}, \bar{\alpha}_{2 i}, \cdots, \bar{\alpha}_{n i}, \cdots\right), \quad i=1,2, \cdots,
$$

and the vector $I_{\pi}$ by

$$
I_{\pi}=\left(\epsilon_{1}, \epsilon_{2}, \cdots, \epsilon_{n}, \cdots\right) .
$$

Introduce the vector space $Q$ as

$$
Q=\left[\zeta_{1}, \zeta_{2}, \cdots\right] \bar{P} .
$$

That is, it is composed of all finite sums $\sum_{i} \zeta_{i} \bar{\alpha}_{i}, \bar{\alpha}_{i}$ in $\bar{P}$. Now (6) can be written as

$$
\sum_{j=1}^{h_{\pi}} \zeta_{j} \bar{\beta}_{\pi j}=I_{\pi}
$$

Thus $I_{\pi}$ is in $Q$. Since $\pi$ is arbitrary, all vectors of the form

$$
I=\left(\epsilon_{1}, \epsilon_{2}, \cdots, \epsilon_{n}, \cdots\right), \quad \epsilon_{n}=0 \text { or } \epsilon_{,}
$$

are in $Q$. That such a conclusion is impossible follows from Lemma 3.1. The assumption that $e^{\theta}\left(\pi_{1}\right), e^{\theta}\left(\pi_{2}\right), \cdots$ is not algebraically summable must be false, and the theorem is established.

A particular instance of this last theorem is $\pi_{n}=(n), n=1,2, \ldots$. Hence the set $e_{11}^{\theta}, e_{22}^{\theta}, \cdots$ is algebraically summable. The endomorphism $\theta$ is not assumed to be complete, so even though we know $e_{11}^{\theta}+e_{22}^{\theta}+\cdots$ is in $\Delta$ and $e_{11}+e_{22}+\cdots=e$, we cannot say that $e^{\theta}=e_{11}^{\theta}+e_{22}^{\theta}+\cdots$ without further justification. Let

$$
\bar{e}=\sum_{i=1}^{\infty} e_{i i}^{\theta}
$$

and let $e^{0}$ be defined by

$$
e^{\theta}=\bar{e}+e^{0}
$$

We shall show that $e^{0}=0$.

From (2.7) and (2.8), $e^{\theta} \bar{e}=\bar{e} e^{\theta}=\bar{e}$. As $\bar{e}$ and $e^{\theta}$ are idempotent, we have

$$
e^{0} e^{0}=e^{0}, \quad e^{0} e^{\theta}=e^{\theta} e^{0}=e^{0}, \quad e^{0} \bar{e}=\bar{e} e^{0}=0 .
$$

Also, as $e_{i j}^{\theta}=e_{i j}^{\theta} \bar{e}=\bar{e} e_{i j}^{\theta}$,

$$
e_{i j}^{0} e^{0}=e^{0} e_{i j}^{\theta}=0, \quad i, j=1,2, \cdots \text {. }
$$

Let $\pi_{1}, \pi_{2}, \cdots$ be any countably infinite partition of the set of integers 
$\rho$ such that all $\pi_{n}$ are infinite sets. Let $\pi_{k}$ be the set of integers $n_{k 1}, n_{k 2}, \ldots$ where $n_{k 1}<n_{k 2}<\cdots, k=1,2, \cdots$. Define

$$
\begin{aligned}
e\left(\pi_{k}\right) & =\sum_{i \in \mathbf{x}} e_{i i}, & k & =1,2, \cdots, \\
e_{k} & =\sum_{i=k}^{\infty} e_{i i}, & k & =1,2, \cdots, \\
p_{k} & =\sum_{i=1}^{\infty} e_{k+i-1 n_{k i},} & k & =1,2, \cdots, \\
q & =\sum_{j=1}^{\infty} \sum_{i=1}^{l} e_{n_{i k} i}, & \text { where } k & =j-i+1 .
\end{aligned}
$$

A simple computation shows that

$$
p_{k} e\left(\pi_{k}\right)=p_{k}, p_{k} e\left(\pi_{k}\right) q=e_{k}, \quad k=1,2, \cdots
$$

From Theorem 3.3 , the set $e^{\theta}\left(\pi_{1}\right), e^{\theta}\left(\pi_{2}\right), \cdots$ is algebraically summable and hence, from Theorem 2.3 , the set $p_{1}^{\theta} e^{\theta}\left(\pi_{1}\right) q^{\theta}, p_{2}^{\theta} e^{\theta}\left(\pi_{2}\right) q^{\theta}, \cdots$ is also algebraically summable. In view of the above equations, $e_{1}^{\theta}, e_{2}^{\theta}, \ldots$ is an algebraically summable set.

Now $e_{1}^{\theta}=e^{\theta}$, and generally

$$
e_{k}^{\theta}=e^{\theta}-\sum_{i=1}^{k-1} e_{i i}^{\theta}, \quad k=2,3, \cdots,
$$

and therefore

$$
e_{k}^{\theta} e^{0}=e^{0} e_{k}^{\theta}=e^{0} e^{\theta}=e^{\theta} e^{0}=e^{0} .
$$

Select any element $\xi$ of $\Xi$. As $e_{1}^{\theta}, e_{2}^{\theta}, \ldots$ is algebraically summable, there exists an integer $N(\xi)$ such that $e_{k}^{\theta} \xi=0, k>N(\xi)$, and consequently

$$
e^{0} e_{k}^{0} \xi=e^{0} \xi=0 \text {. }
$$

It follows that $e^{0 \xi}=0$ for all $\xi$ in $\Xi$ so that $e^{0}=0$. This establishes the following lemma.

LEMMA 3.2. If $\theta$ is an endomorphism of $\Delta$, then $e_{11}^{\theta}, e_{22}^{\theta}, \cdots$ is algebraically summable and

$$
e^{\theta}=\dot{e}_{11}^{\theta}+\dot{e}_{22}+\cdots .
$$

Consider any algebraically summable set $a_{1}, a_{2}, \cdots$ of elements of $\Delta$, and let $a=a_{1}+a_{2}+\cdots$. By definition, there exist integers $N_{1}, N_{2}, \cdots$ such that

$$
a_{n} \xi_{k}=0, \quad n>N_{k}, k=1,2, \cdots .
$$


An alternate way of writing this equation is

$$
a_{n} e_{k k}=0, \quad n>N_{k}, k=1,2, \ldots .
$$

In view of the preceding lemma, for any $\xi$ in $\Xi$ there exists an integer $N(\xi)$ such that

$$
\dot{e}_{n n}^{\dot{\xi}} \xi=0, \quad n>N(\xi),
$$

and therefore

$$
a_{k}^{\theta} \xi=\left(a_{k}^{0} e_{11}^{\theta}+a_{k}^{\theta} e_{22}^{\theta}+\cdots+a_{k}^{0} e_{n n}^{\theta}\right) \xi, \quad n=N(\xi), k=1,2, \cdots .
$$

Select the integer $M$ as

$$
M=\max \left(N_{1}, N_{2}, \cdots, N_{n}\right), \quad n=N(\xi) .
$$

Then

$$
a_{k}^{\theta} e_{i i}^{0}=0, \quad k>M, i=1,2, \cdots, N(\xi),
$$

so that

$$
\boldsymbol{a}_{k}^{0} \xi=0, \quad k>M,
$$

and $a_{1}, a_{2}, \cdots$ is algebraically summable. Further,

$$
a e_{k k}=a_{1} e_{k k}+a_{2} e_{k k}+\cdots+a_{n} e_{k k}, \quad n=N_{k}, k=1,2, \cdots,
$$

so that

$a^{\theta} e_{k k}^{\theta}=a_{1}^{\theta} e_{k k}^{\theta}+a_{2}^{\theta} e_{k k}^{\theta}+\cdots+a_{n}^{\theta} e_{k k}^{\theta}=\left(a_{1}^{\theta}+a_{2}^{\theta}+\cdots\right) e_{k k}^{\theta}, \quad k=1,2, \cdots$.

If we sum up both sides of this equation with respect to $k$,

$$
a^{0}=a_{1}^{0}+a_{2}^{0}+\cdots \text {. }
$$

We have established the following theorem.

THEOREM 3.4. Every endomorphism of $\Delta$ is a complete endomorphism( ${ }^{(4)}$.

If $\theta$ is a proper endomorphism, then $\theta$ is a proper complete endomorphism so that $\theta$ is the zero endomorphism because of Theorem 3.2. Hence we have the following corollary.

COROLLARY. Every nonzero endomorphism of $\Delta$ is a complete meromorphism.

4. Determination of all meromorphisms of $\Delta$. Consider any meromorphism $\theta$ of $\Delta$ into $\Delta^{\theta}$. By the preceding section, $\theta$ is a complete meromorphism. Let

(4) If we introduce the weak topology in $\Delta$ we convert $\Delta$ into a topological ring and it is easy to see that algebraic summability coincides with convergence in this topology. Theorem 3.4 can thus be stated as the result that every endomorphism of $\Delta$ is continuous in the weak topology. (This remark is due to the referee.) 


$$
\Xi^{\theta}=e^{\theta} \Xi, \quad H^{\theta}=H_{e^{\theta},}
$$

that is, $\Xi^{\theta}$ is the set of all $e^{\theta} \xi$ while $H^{\theta}$ is the set of all $\xi$ annihilated by $e^{\theta}, \xi$ in $\Xi$. From the Peirce decomposition,

$$
\Xi=\Xi^{\theta}+H^{\theta} .
$$

As $a^{\theta} \xi=e^{\theta}\left(a^{\theta} \xi\right), a^{\theta} \xi$ is in $\Xi^{\theta}$, while if $e^{\theta} \xi=0$ then $a^{\theta} \xi=0$, and therefore both $\Xi^{\theta}$ and $H^{\theta}$ are $\Delta^{\theta}$-modules. In the work to follow, we shall use the basis of $\Xi$ given in (1.1): $:=P \xi_{1}+P \xi_{2}+\cdots$, and the corresponding basis elements of $\Delta$, the $e_{i j}$ defined in (2.2).

Let $\xi$ be any nonzero element of $\boldsymbol{\Xi}^{\theta}$. As $e^{\theta} \xi=\xi$, and in view of Lemma 3.2, there exists an integer $n$ such that $e_{n n}^{\theta} \xi \neq 0$. Define

$$
\eta_{n}=e_{n n}^{\theta} ; \quad \eta_{i}=e_{i n}^{\theta} \eta_{n}, \quad i=1,2, \cdots .
$$

From (2.3) it is evident that

$$
\dot{e}_{i j}^{\bullet} \eta_{j}=\eta_{i} ; \quad \dot{e}_{i j}^{\bullet} \eta_{k}=0, \quad j \neq k ; i, j, k=1,2, \cdots .
$$

And as $\eta_{n}=e_{m i}^{\theta} \eta_{i}$, all $\eta_{i}$ must be different from zero. If

$$
\sum_{i=1}^{m} \alpha_{i} \eta_{i}=0
$$

$\alpha_{i}$ in $P$,

then

$$
e_{r s} \sum_{i=1}^{m} \alpha_{i} \eta_{i}=\alpha_{s} \eta_{r}=0, \quad r, s=1,2, \cdots, m .
$$

This is possible only if $\alpha_{s}=0, s=1,2, \cdots, m$, so that the following lemma is established.

LemMa 4.1. A set of nonzero elements $\eta_{1}, \eta_{2}, \ldots$ of $\Xi^{\theta}$ satisfying (4.1) is necessarily linearly independent.

The division rings $P$ and $\bar{P}$ have a common center $C$. The set of all elements of $\Delta$ of the form

$$
\sum_{j=1}^{\infty} \sum_{i=1}^{n_{j}} \alpha_{i j} e_{i j}^{0}
$$

$\alpha_{i j}$ in $C$,

with the operations of $\Delta$ is a ring $R$. In case $P$ is a field and $\theta$ is a $P$-endomorphism $R$ is just $\Delta^{\theta}$.

If a subset $\Xi_{1}$ of $\Xi$ is both a $P$-module and an $R$-module, $\Xi_{1}$ is called a $(P, R)$-module. Any nonzero $(P, R)$-module, $\Xi_{1}$, is a minimal $(P, R)$-module if no proper nonzero submodule of $\Xi_{1}$ is a $(P, R)$-module. As an example of a minimal $(P, R)$-module, let the nonzero elements $\eta_{1}, \eta_{2}, \cdots$ of $\Xi$ have property (4.1). Then $\Xi_{1}=P \eta_{1}+P \eta_{2}+\cdots$ is a minimal $(P, R)$-module. It is 
not in general true that $\Xi_{1}$ is a $\Delta^{\theta}$-module, for $\bar{\alpha}^{\theta} \eta_{i}$ need not be in $\Xi_{1}$. An example to be given later will illustrate this point. It is evident that every minimal $(P, R)$-module $\Xi_{1}$ has a proper $P$-basis $\eta_{1}, \eta_{2}, \cdots$ satisfying $(4.1)$, as the element $\xi$ in the paragraph preceding Lemma 4.1 could be selected from $\mathbf{\Xi}_{1}$.

TheOREM 4.1. The module $\Xi^{\theta}$ has a decomposition into a direct sum

$$
\Xi^{\theta}=\Xi_{1}+\Xi_{2}+\cdots
$$

of minimal $(P, R)$-modules. Each $\Xi_{k}$ has a representation

$$
\Xi_{k}=P \eta_{1 k}+P \eta_{2 k}+\cdots
$$

with the property

$$
\stackrel{\theta}{e_{i j} \eta_{j k}}=\eta_{i k} ; \quad{\stackrel{\theta}{e_{i} \eta_{n k}}}_{n}=0, \quad j \neq n ; i, j, k, n=1,2, \cdots,
$$

and $\eta_{j k}=e_{j m}^{\theta} \xi_{n}$ for some choice of $m$ and $n$.

Proof. To any integer $k$ there corresponds an integer $N_{k}$ such that $e_{n n}^{\theta} \xi_{k}=0, n>N_{k}$, because of Lemma 3.2. Thus $e^{\theta} \xi_{k}=e_{11}^{\theta} \xi_{k}+e_{22}^{\theta} \xi_{k}+\cdots$ $+e_{m m}^{\theta} \xi_{k}, m=N_{k}$. Define

As

$$
\begin{aligned}
\eta_{\text {ink }} & =e_{i n} \xi_{k}, & i, n, k & =1,2, \cdots, \\
\Xi_{n k} & =P \eta_{1 n k}+P \eta_{2 n k}+\cdots, & n, k & =1,2, \cdots .
\end{aligned}
$$

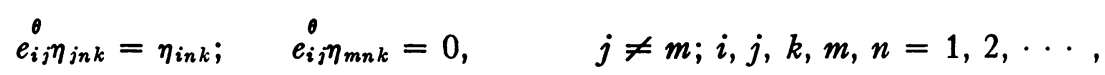

each $\Xi_{n k}$ is either zero or a minimal $(P, R)$-module. The element $e^{\theta} \xi_{k}$ is in $\left(\boldsymbol{\Xi}_{1 k}, \boldsymbol{\Xi}_{2 k}, \cdots, \boldsymbol{\Xi}_{n k}\right), n=N_{k}$, and therefore $\boldsymbol{\Xi}^{\theta}=\mathrm{U}\left(\boldsymbol{\Xi}_{n k}\right), n, k=1,2, \cdots$ Any one of the usual methods of eliminating the dependent $\Xi_{n k}$ will yield a decomposition such as (4.2).

Lemma 4.2. The number of minimal $(P, R)$-modules in any decomposition (4.2) of $\mathbf{Z}^{\theta}$ is an invariant of $\theta$.

Proof. If $\boldsymbol{\Xi}^{\theta}=\boldsymbol{\Xi}_{1}+\boldsymbol{\Xi}_{2}+\cdots=\boldsymbol{\Xi}_{1}^{\prime}+\boldsymbol{\Xi}_{2}^{\prime}+\cdots$ with each $\boldsymbol{\Xi}_{n}, \boldsymbol{\Xi}_{m}^{\prime}$ a minimal $(P, R)$-module and

$$
\begin{aligned}
& \Xi_{k}=P \eta_{1 k}+P \eta_{2 k}+\cdots, & k & =1,2, \cdots, \\
& \Xi_{k}^{\prime}=P \eta_{1 k}^{\prime}+P \eta_{2 k}^{\prime}+\cdots, & k & =1,2, \cdots, \\
\boldsymbol{e}_{i j}^{\prime} \eta_{j k}=\eta_{i k} ; & e_{i j}^{\theta} \eta_{n k}=0, & j \neq n ; i, j, k, n & =1,2, \cdots, \\
e_{i j}^{\prime} \eta_{j k}^{\prime}=\eta_{i k}^{\prime} ; & e_{i j}^{\theta} \eta_{n k}^{\prime}=0, & j \neq n ; i, j, k, n & =1,2, \cdots,
\end{aligned}
$$

then

$$
\stackrel{\theta}{e}_{11}^{\theta} \Xi^{\theta}=P \eta_{11}+P \eta_{12}+\cdots=P \eta_{11}^{\prime}+P \eta_{22}^{\prime}+\cdots,
$$


the order of a $P$-module, that is, the number of elements in a proper $P$-basis is an invariant of the $P$-module. The above equation shows that the number of $\eta_{1 i}$ equals the number of $\eta_{1 i}^{\prime}$, and therefore the lemma follows.

Definition 4.1. The number of minimal $(P, R)$-modules in a decomposition (such as (4.2)) of $\boldsymbol{\Xi}^{\theta}$ is called the order of $\theta, o(\theta)$, and is a positive integer or $\aleph_{0}$.

Let us now assume that $\Xi^{\theta}$ has the decomposition (4.2) into a direct sum of minimal $(P, R)$-modules $\boldsymbol{\Xi}_{k}$, and each $\boldsymbol{\Xi}_{k}$ has the basis (4.3) with property (4.4). As $\Xi=\Xi^{\theta}+H^{\theta}$, and because of Theorem 1.1 , there exist unique elements $u_{1}, u_{2}, \cdots, v_{1}, v_{2}, \cdots$ of $\Delta$ defined by

$$
\begin{aligned}
& u_{k} \eta_{i k}=\xi_{i} ; \quad u_{k} \eta_{i j}=0, \quad j \neq k ; u_{k} H^{\theta}=0, i, j, k=1,2, \cdots, \\
& v_{k} \xi_{i}=\eta_{i k}, \quad i, k=1,2, \cdots \text {. }
\end{aligned}
$$

It is evident that $u_{k}$ and $v_{k}$ are semi-inverses of each other. In fact

$$
u_{k} v_{k}=e ; \quad u_{k} v_{j}=0, \quad j \neq k ; j, k=1,2, \cdots .
$$

The set $u_{1}, u_{2}, \cdots$ is algebraically summable as $u_{k} \eta_{i n}=0, k>n$, and $u_{k} H^{\theta}=0$. In view of Theorem $2.3, v_{1} u_{1}, v_{2} u_{2}, \cdots$ is also an algebraically summable set, and

$$
\begin{aligned}
& \left(v_{1} u_{1}+v_{2} u_{2}+\cdots\right) \eta_{n m}=\eta_{n m}, \quad n, m=1,2, \cdots, \\
& \left(v_{1} u_{1}+v_{2} u_{2}+\cdots\right) H^{\theta}=0 .
\end{aligned}
$$

However $e^{\theta}$ has exactly the same effect on this basis: $e^{\theta} \eta_{n m}=\eta_{n m}, e^{\theta} H^{\theta}=0$; so as a consequence

$$
e^{\theta}=v_{1} u_{1}+v_{2} u_{2}+\cdots .
$$

From (2.2), (4.4), and (4.5) we derive

$$
\begin{aligned}
e_{i j}^{\theta} v_{k} \xi_{j} & =\eta_{i k}=v_{k} \xi_{i}=v_{k} e_{i j} \xi_{j} ; & i, j, k & =1,2, \cdots, \\
e_{i j}^{\theta} v_{k} \xi_{n} & =v_{k} e_{i j} \xi_{n}=0, & n \neq j ; i, j, k, n & =1,2, \cdots .
\end{aligned}
$$

And therefore

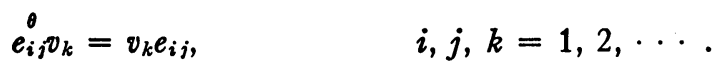

From this we obtain $e_{i j}^{\theta} v_{k} u_{k}=v_{k} e_{i j} u_{k}$, so that the use of (4.7) yields

$$
e_{i j}^{\theta}=\sum_{k} v_{k} e_{i j} u_{k}, \quad i, j=1,2, \cdots,
$$

the summation going from 1 to $o(\theta)$.

We recall that corresponding to the basis (1.1), $\Xi=P \xi_{1}+P \xi_{2}+\cdots$, there is a division subring $\bar{P}$ of $\Delta$ anti-isomorphic to $P ; \bar{\alpha}$ in $\bar{P}$ is defined by $\bar{\alpha} \xi_{i}$ $=\alpha \xi_{i}, i=1,2, \ldots$ Under the meromorphism $\theta, \bar{P}$ is carried into $\bar{P}^{\theta}$.

For any $\bar{\alpha}^{\theta}$ in $\bar{P}^{\theta}$ and $\xi$ in $\Xi^{\theta}, \bar{\alpha}^{\theta} \xi$ is also in $\Xi^{\theta}$. Hence 


$$
\bar{\alpha}^{0} \eta_{r s}=\sum_{i, j} \gamma_{i j r s} \eta_{i j}, \quad r, s=1,2, \cdots
$$

Now $e_{i j}^{\theta} \bar{\alpha}^{\theta}=\bar{\alpha}^{\theta} e_{i j}^{\theta}$, so that, on multiplying this equation by $e_{k r}^{\theta}$, we obtain

$$
\bar{\alpha}^{\theta} \eta_{k s}=\sum_{i} \gamma_{r j r s} \eta_{k j}, \quad r, s, k=1,2, \cdots
$$

Thus the $\gamma$ 's are independent of the $r$ in $\eta_{r s .}$ However, the $\gamma$ 's are functions of $\alpha$, and to show this we rewrite the equation as

$$
\dot{\alpha}^{0} \eta_{r s}=\sum_{i} \rho_{i s}^{\alpha} \eta_{r i}, \quad r, s=1,2, \cdots .
$$

In this equation $\rho_{s}^{\alpha}=0$ if $i$ is larger than some integer which is a function of $s$ and $\alpha$.

Similarly, for any $\beta$ in $P$,

$$
\widehat{\beta}_{\eta_{r e}}=\sum_{i} \rho_{i s}^{\beta} \eta_{r i}
$$

Then

$$
\bar{\beta}^{0} \bar{\alpha}^{\theta} \eta_{r s}=\sum_{i} \rho_{i s}^{\alpha} \sum_{i} \stackrel{\beta}{\rho_{j i} \eta_{r j}}
$$

On the other hand,

$$
\overline{\alpha \beta}^{0} \eta_{r s}=\sum_{i} \rho_{i s}^{\alpha \beta} \eta_{r i}
$$

and as $\overline{\alpha \beta^{\theta}}=\bar{\beta}^{\ominus} \bar{\alpha}^{\theta}$, we must have

$$
\rho_{r \varepsilon}^{\alpha \beta}=\sum_{i} \stackrel{\alpha \beta}{\rho_{i s}^{\beta} \rho_{r i}}
$$

The corresponding equation in the division ring $\bar{P}$ is

$$
\bar{\rho}_{r e}^{\alpha \beta}=\sum_{i} \bar{\rho}_{r i}^{\beta} \bar{\rho}_{i s}^{\alpha} .
$$

Evidently

$$
\bar{\rho}_{r s}^{\alpha+\beta}=\bar{\rho}_{r s}^{\alpha}+\bar{\rho}_{r s}^{\beta}
$$

also holds.

The correspondence

$$
\bar{\alpha} \rightarrow f_{\alpha}=\sum_{r, s} \bar{p}_{r s}^{\alpha} e_{r s},
$$

where $r$ is finite for each $s$ and $s$ has $o(\theta)$ for its range, is an isomorphism between $\bar{P}$ and a subring of $\Delta$. For from (4.11) and (4.12) we have

$$
f_{\alpha \beta}=f_{\beta} f_{\alpha}, \quad f_{\alpha+\beta}=f_{\alpha}+f_{\beta},
$$


and as $0^{\theta}=0, f_{\alpha}=0$ if and only if $\bar{\alpha}=0$. Now $P$ and $\bar{P}$ are anti-isomorphic under the correspondence $\alpha \leftrightarrow \bar{\alpha}$, so these equations show that $\bar{\alpha} \leftrightarrow f_{\alpha}$ is an isomorphism between $\bar{P}$ and a subring of $\Delta$.

With the aid of $\left(1.3^{\prime}\right),(4.5)$, and $(4.10)$, remembering that $\rho_{t r}^{\alpha} \xi_{r}=\bar{p}_{t s}^{\alpha} \xi_{r}$, we obtain

$$
\bar{\alpha}^{\theta} v_{s} \xi_{r}=\bar{\alpha}^{\theta} \eta_{r s}=\sum_{i} \rho_{i s}^{\alpha} v_{i} \xi_{r}=\sum_{i} v_{i} \bar{\rho}_{i s}^{\alpha} \xi_{r}
$$

and therefore

$$
\bar{\alpha}^{0} v_{s}=\sum_{i} v_{i} \bar{\rho}_{i s}^{\alpha}
$$

Using the same methods employed in deriving (4.9) from (4.8), we obtain finally

$$
\bar{\alpha}^{0}=\sum_{r, s} v_{r} \tilde{\rho}_{r s}^{*} u_{s} .
$$

In this summation, $r$ has a finite range for each value of $s$ and $s$ has $o(\theta)$ as its range.

We shall now combine (4.9) and (4.14) to get an explicit formulation of $a^{\theta}$ for any $a$ in $\Delta$. Let $a$, have the representation

$$
a=\sum_{i, j} \bar{\alpha}_{i j} e_{i j}
$$

As $\theta$ is complete,

$$
a^{0}=\sum_{i, j} \dot{\alpha}_{i j}^{0} e_{i j}^{0}
$$

In view of (4.9) and (4.14),

$$
a^{\theta}=\sum_{r, e} v_{r}\left(\sum_{i, j}^{\alpha_{r_{i e}}^{\alpha_{i j}} e_{i j}}\right) u_{s e}
$$

We have established the following theorem.

THEOREM 4.2. If $\theta$ is a meromorphism of $\Delta$, there exists an algebraically summable set of elements $u_{1}, u_{2}, \cdots, o(\theta)$ in number and a corresponding set $v_{1}, v_{2}, \cdots$ with the property

$$
u_{k} v_{k}=e, \quad u_{k} v_{j}=0, \quad j \neq k ; j, k=1,2, \cdots, o(\theta),
$$

and an isomorphism between $\bar{P}$ and a subring of $\Delta, \bar{\alpha} \leftrightarrow f_{\alpha}=\sum \bar{\rho}_{r s}^{\alpha} e_{r s}$, such that for any element $a$ of $\Delta$ given by $a=\sum \bar{\alpha}_{i j} e_{i j}$,

$$
a^{\prime}=\sum_{r, s} v_{r}\left(\sum_{i, j} \bar{\rho}_{r s}^{\alpha_{i j}} e_{i j}\right) u_{s \cdot}
$$


The converse of this theorem takes the following form:

THEOREM 4.3. If the set $u_{1}, u_{2}, \cdots, u_{N}, N$ a positive integer or $\boldsymbol{\aleph}_{0}$, is algebraically summable, and if there exist elements $v_{1}, v_{2}, \cdots, v_{N}$ for which

$$
u_{k} v_{k}=e, \quad u_{k} v_{j}=0, \quad j \neq k ; j, k=1,2, \cdots, N,
$$

and if

$$
\bar{\alpha} \leftrightarrow f_{\alpha}=\sum_{r, s=1}^{N} \bar{\rho}_{r s}^{\alpha} e_{r s}
$$

is an isomorphism between $\bar{P}$ and a subring of $\Delta$, then for $a=\sum \bar{\alpha}_{i j} e_{i j}$ the correspondence $\theta$ defined by

$$
a^{\theta}=\sum_{r, s=1}^{N} v_{r}\left(\sum_{i, j} \bar{\rho}_{r s}^{\alpha_{i j}} e_{i j}\right) u_{s}
$$

is a meromorphism of $\Delta$.

Proof. It is apparent that $a^{\theta}+b^{\theta}=(a+b)^{\theta}$. Also, if $a^{\theta}=0$, then $u_{r} a^{\theta} v_{s}=0$ so that

$$
\bar{\rho}_{r s}^{\alpha_{i j}}=0: \quad r, s=1,2, \cdots, N ; i, j=1,2, \cdots ;
$$

then

$$
f_{\alpha_{i j}}=0, \quad i, j=1,2, \cdots,
$$

and $\alpha_{i j}=0$ for all $i, j$. Hence $a=0$.

If $b=\sum \bar{\beta}_{i j} e_{i j}$,

$$
a^{\theta} b^{\theta}=\sum_{r, s, t=1}^{N} v_{r}\left(\sum_{i, j, k} \bar{\rho}_{r s}^{\alpha_{i j} \bar{\rho}_{s t} \beta_{i j k}}\right) u_{t \cdot}
$$

Calling $a b=\sum \bar{\gamma}_{i j} e_{i j}$ and remembering that

$$
\sum_{s=1}^{N} \bar{\rho}_{r s}^{\alpha} \bar{\rho}_{s t}^{\beta}=\bar{\rho}_{r t}^{\beta \alpha}
$$

then we have

$$
a^{0} b^{\theta}=\sum_{r, s=1}^{N} v_{r}\left(\sum_{i, j, k} \bar{\rho}_{r s}^{\beta_{j k} \alpha_{i j}} e_{i k}\right) u_{s},
$$

and as $\bar{\gamma}_{i j}=\sum_{k} \bar{\alpha}_{i k} \bar{\beta}_{k j}=\sum_{k} \beta_{k j} \alpha_{i k}$,

$$
a^{\theta} b^{\theta}=\sum_{r, s=1}^{N} v_{r}\left(\sum_{i, j} \bar{\rho}_{r s}^{\gamma_{i j}} e_{i j}\right) u_{s}
$$

This establishes the theorem. 
The above representation assumes a simple form in case $\theta$ is an automorphism. As $u_{1}, v_{1}$ are not zero, there exists a $b$ in $\Delta, b=\sum \bar{\beta}_{i j} e_{i j}$, such that $v_{1} u_{1}=b^{\theta} \neq 0$. By Theorem 4.2

$$
v_{1} u_{1}=\sum_{r, 8} v_{r}\left(\sum_{i, j} \bar{\rho}_{r s}^{\beta_{i j}} e_{i j}\right) u_{s .}
$$

From (4.6) we derive, if $o(\theta)>1$,

$$
\begin{array}{rlrl}
u_{1}\left(v_{1} u_{1}\right) v_{1} & =\sum_{i, j} \bar{\rho}_{11}^{\beta_{i j}} e_{i j}=e, & \\
u_{k}\left(v_{1} u_{1}\right) v_{1} & =\sum_{i, j} \bar{\rho}_{k 1}^{\beta_{i j}} e_{i j}=0, & k>1, \\
u_{1}\left(v_{1} u_{1}\right) v_{k}=\sum_{i, j} \bar{\rho}_{1 k}^{\beta_{i j}} e_{i j}=0, & k>1 .
\end{array}
$$

Hence

$$
\begin{aligned}
& \bar{\rho}_{11}^{\beta_{i j}}=\bar{\epsilon} \text { if } i=j ; \quad \overline{\boldsymbol{\rho}}_{11}^{\beta_{i j}}=0 \text { if } i \neq j . \\
& \bar{\rho}_{r s}^{\beta_{i j}}=0 \quad \text { if } r+s>2 .
\end{aligned}
$$

A glance at (4.13) reveals that

$$
\bar{\beta}_{i j}=0, \quad i \neq j ; \quad \bar{\beta}_{i i}=\bar{\epsilon} .
$$

Thus $v_{1} u_{1}=e^{\theta}$ and consequently $v_{k} u_{k}=0, k>1$. The order of $\theta$ is one and Theorem 4.2 becomes $\left.{ }^{5}\right)$ :

THEOREM 4.4. If $\theta$ is an automorphism of $\Delta$, there exists an automorphism $\bar{\alpha} \leftrightarrow \overline{\boldsymbol{\rho}}^{\alpha}$ of $\bar{P}$ and a regular element $u$ of $\Delta$ such that, if $a=\sum \alpha_{i j} e_{i j}$, then

$$
a^{\theta}=u^{-1}\left(\sum \bar{\rho}^{\alpha_{i j}} e_{i j}\right) u \text {. }
$$

As an example of the above, take $P$ as rational quaternions, $P=R_{a}(1, i, j, k)$. Let $\Xi$ be the linear set of all vectors $\left(\alpha_{1}, \alpha_{2}, \cdots\right)$ over $P$ of order type $\omega$ with only a finite number of nonzero coordinates. Then $\Delta$ is the ring of all $\omega \times \omega$ matrices $\left(\bar{\alpha}_{r s}: r, s=1,2, \cdots\right)$ over $\bar{P}$, a ring antiisomorphic to $P$, with finitely nonzero columns (that is, $\bar{\alpha}_{r s}=0$ for $r>N_{s}, N_{s}$ an integer depending on $s$ ).

Select $\xi_{k}$ as the vector with 1 as the $k$ th coordinate and 0 's elsewhere. Then

$$
\Xi=P \xi_{1}+P \xi_{2}+\cdots \text {. }
$$

For this basis of $\Xi, e_{i j}$ is the matrix with 1 in the $i$ th row and $j$ th column and 0 's elsewhere.

(5) This theorem is a special case of a theorem given by N. Jacobson for dense rings. See N. Jacobson, The radical and semi-simplicity for arbitrary rings, Amer. J. Math. vol. 47 (1945) p. 318, Theorem 32. 
For $\alpha$ in $P$, the correspondence

$$
\bar{\alpha} \leftrightarrow-j \bar{\alpha} \bar{j} e_{11}+(j \bar{\alpha} \bar{z}-\bar{\alpha} \bar{k}) e_{12}-\bar{k} \bar{\alpha} \bar{k} e_{22}
$$

is an isomorphism between $\bar{P}$ and a subring of $\Delta$. Let

$$
u_{1}=\sum_{i=1}^{\infty} e_{i 2 i-1}, \quad u_{2}=\sum_{i=1}^{\infty} e_{i 2 i}, \quad v_{1}=\sum_{i=1}^{\infty} e_{2 i-1 i}, \quad v_{2}=\sum_{i=1}^{\infty} e_{2 i} \text {. }
$$

Then

$$
u_{1} v_{1}=u_{2} v_{2}=e ; \quad u_{1} v_{2}=u_{2} v_{1}=0
$$

in accordance with (4.6). For the matrix $a$ in $\Delta$, the correspondence $\theta$ defined by

$$
a^{\theta}=-v_{1} \bar{j} a \bar{j} u_{1}+v_{1}(\bar{j} a \bar{z}-a \bar{k}) u_{2}-v_{2} \bar{k} a \bar{k} v_{2}
$$

is a meromorphism of $\Delta$ as a consequence of Theorem 4.3. The order of $\theta$, $o(\theta)$, equals 2 , as

$$
\Xi_{1}=P \xi_{1}+P \xi_{3}+\cdots, \quad \Xi_{2}=P \xi_{2}+P \xi_{4}+\cdots,
$$

and

$$
\Xi=\Xi^{0}=\Xi_{1}+\Xi_{2}
$$

with $\Xi_{1}$ and $\boldsymbol{Z}_{2}$ minimal $(P, R)$-modules.

5. The $\bar{P}$-meromorphisms of $\Delta$. Theorem 2.3 indicates the importance of the division ring $\bar{P}$ in a given representation of $\Delta$ as a matrix algebra. The meromorphism $\theta$ of $\Delta$ is a $\bar{P}$-meromorphism if for $a$ in $\Delta, \bar{\alpha}$ in $\bar{P}$,

$$
(\bar{\alpha} a)^{\theta}=\bar{\alpha} a^{\theta}, \quad(a \bar{\alpha})^{\theta}=a^{\theta} \bar{\alpha} .
$$

An equivalent condition is that

$$
\bar{\alpha}^{\theta}=\bar{\alpha} e^{\theta}=e^{\theta} \bar{\alpha} .
$$

If $\theta$ is a $\bar{P}$-meromorphism, then by Theorem 4.1 there exists a representation of $\Xi^{\theta}$ as a sum of minimal $(P, R)$-modules:

$$
\Xi^{0}=\Xi_{1}+\Xi_{2}+\cdots
$$

with

$$
\Xi_{k}=P \eta_{1 k}+P \eta_{2 k}+\cdots
$$

and

$$
\dot{e}_{i j} \eta_{j k}=\eta_{i k} ; \quad \dot{e}_{i j} \eta_{n k}=0, \quad j \neq n \text {. }
$$

Furthermore, $\eta_{j k}=e_{m n}^{\theta} \xi_{m}$ for some choice of $m$ and $n$ depending on $j$ and $k$. Hence 


$$
\bar{\alpha}^{\theta} \eta_{j k}=\bar{\alpha}^{\theta} e_{j n}^{\theta} \xi_{m}=e_{j n}^{\theta} \bar{\alpha} \xi_{m}=e_{j n}^{\theta} e^{\theta} \bar{\alpha} \xi_{m}=\alpha_{j n}^{\theta} \alpha \xi_{m}=\alpha e_{j n}^{\theta} \xi_{m}=\alpha \eta_{j k}
$$

for $j, k=1,2, \cdots$ and any $\alpha$ in $P$. From (4.10),

$$
\stackrel{\alpha}{\rho_{j i}}=\alpha ; \quad \stackrel{\alpha}{\rho_{j k}}=0, \quad j \neq k .
$$

Theorem 4.2 then takes the following form:

THEOREM 5.1. If $\theta$ is a $\bar{P}$-meromorphism of $\Delta$ of order $o(\theta)$, there exists an algebraically summable set of elements $u_{1}, u_{2}, \cdots, o(\theta)$ in number, and a corresponding set $v_{1}, v_{2}, \cdots$ with the property

$$
u_{k} v_{k}=e ; \quad u_{k} v_{j}=0, \quad j \neq k ; j, k=1,2, \cdots, o(\theta)
$$

such that

$$
a^{0}=\sum_{k} v_{k} a u_{k}
$$

Evidently

$$
a^{\theta} v_{k}=v_{k} a ; \quad u_{k} a^{\theta}=a u_{k}, \quad k=1,2, \cdots,
$$

and as

$$
\bar{\alpha}^{\theta} v_{k}=\bar{\alpha} e^{\theta} v_{k}=\bar{\alpha} v_{k}, \quad u_{k} \bar{\alpha}^{\theta}=u_{k} e^{\theta} \bar{\alpha}=u_{k} \bar{\alpha},
$$

it follows that

$$
\bar{\alpha} v_{k}=v_{k} \bar{\alpha}, \quad \bar{\alpha} u_{k}=u_{k} \bar{\alpha}, \quad k=1,2, \ldots .
$$

In other words, the ring $\bar{P}$ commutes elementwise with the $u_{k}$ and $v_{k}$.

Let $\theta_{k}$ be the inner meromorphism defined by

$$
a^{\theta_{k}}=v_{k} a u_{k} \text {. }
$$

From (5.2), $\theta_{k}$ is a $\bar{P}$-meromorphism. The meromorphisms $\theta_{1}, \theta_{2}, \cdots$ are orthogonal and the set $e^{\theta_{1}}, e^{\theta_{2}}, \cdots$ is algebraically summable. We conclude that the following theorem holds.

TheOREM 5.2. A meromorphism $\theta$ is a $\bar{P}$-meromorphism if and only if there exists a set of inner $\bar{P}$-meromorphisms $\theta_{1}, \theta_{2}, \cdots$ containing $o(\theta)$ elements such that

$$
\theta=\theta_{1} \oplus \theta_{2} \oplus \cdots
$$

Corollary. If $\theta$ is a $\bar{P}$-automorphism of $\Delta$, then $\theta$ is an inner automorphism.

If $\theta$ and $\phi$ are meromorphisms of $\Delta$, then the product of $\theta$ and $\phi, \theta \phi$, defined by $a^{\theta \phi}=\left(a^{\theta}\right)^{\phi}$, is also a meromorphism of $\Delta$. If $\theta$ and $\phi$ are $\bar{P}$-meromorphisms, $\theta \phi$ is also a $\bar{P}$-meromorphism.

Theorem 5.3. If $\theta$ is a $\bar{P}$-meromorphism of $\Delta$ and if $\theta$ has the two decom- 
positions

$$
\theta=\theta_{1} \oplus \theta_{2} \oplus \cdots=\phi_{1} \oplus \phi_{2} \oplus \cdots
$$

into direct sums of inner $\bar{P}$-meromorphisms, then the sets $\theta_{1}, \theta_{2}, \cdots$ and $\phi_{1}$, $\phi_{2}, \cdots$ have the same number of elements, $o(\theta)$, and there exists a $\bar{P}$-automorphism $\tau$ of $\Delta$ such that

$$
\phi_{i}=\theta_{i} \tau, \quad i=1,2, \cdots .
$$

Proof. As $\theta_{i}$ and $\phi_{j}$ are inner $\bar{P}$-meromorphisms,

$$
\begin{aligned}
a^{\theta_{i}} & =v_{i} a u_{i}, & i & =1,2, \cdots, \\
a^{\phi_{i}} & =t_{j} a s_{j}, & j & =1,2, \cdots,
\end{aligned}
$$

and

$$
\begin{aligned}
u_{i} v_{i} & =e, \\
s_{j} t_{j} & =e,
\end{aligned}
$$$$
i=1,2, \cdots,
$$$$
j=1,2, \cdots \text {. }
$$

Now $e^{\theta_{i}} e^{\theta_{j}}=0$ if $i \neq j$ and similarly for $\phi_{j}$, so that

$$
\begin{aligned}
u_{i} v_{j}=0, & i \neq j, \\
s_{i} t_{j}=0, & i \neq j .
\end{aligned}
$$

Any element $\bar{\alpha}$ of $\bar{P}$ commutes with all $u_{i}, v_{i}, s_{j}, t_{j}$. Also

$$
e^{\theta}=v_{1} u_{1}+v_{2} u_{2}+\cdots=t_{1} s_{1}+t_{2} s_{2}+\cdots .
$$

Let $\zeta_{i k}$ and $\eta_{i k}$ of $\Xi$ be defined by $\zeta_{i k}=v_{k} \xi_{i} ; \eta_{i k}=t_{k} \xi_{i}, i, k=1,2, \cdots$, from which it follows that

$$
\begin{array}{rlrl}
u_{k} \zeta_{i k} & =\xi_{i} ; & u_{k} \zeta_{i j}=0, & j \neq k, \\
s_{k} \eta_{i k}=\xi_{i} ; & s_{k} \eta_{i j}=0, & j \neq k .
\end{array}
$$

It is also seen that for any $\alpha$ in $P$,

$$
\alpha \zeta_{i k}=\bar{\alpha} \zeta_{i k} ; \quad \alpha \eta_{i k}=\bar{\alpha} \eta_{i k}, \quad i, k=1,2, \cdots .
$$

If $\Xi_{k}$ and $\Pi_{j}$ be defined by

$$
\Xi_{k}=e^{\theta_{k}} \Xi, \quad \Pi_{j}=e^{\phi_{j}} \Xi,
$$

then

$$
\Xi^{0}=\Xi_{1}+\Xi_{2}+\cdots=\Pi_{1}+\Pi_{2}+\cdots
$$

Furthermore,

$$
\begin{aligned}
& \Xi_{k}=P \zeta_{1 k}+P \zeta_{2 k}+\cdots, \\
& k=1,2, \cdots, \\
& \Pi_{j}=P \eta_{1 j}+P \eta_{2 j}+\cdots \text {, } \\
& j=1,2, \cdots \text {, }
\end{aligned}
$$


and

$$
\begin{aligned}
& {\stackrel{\theta}{e_{i j}} \zeta_{j k}}=\zeta_{i k} ; \quad \stackrel{\theta}{e}_{i j} \zeta_{n k}=0, \\
& j \neq n \text {, } \\
& \stackrel{\theta}{e_{i j} \eta_{i k}}=\eta_{i k} ; \quad \dot{e}_{i j}^{\theta} \eta_{n k}=0, \\
& j \neq n \text {. }
\end{aligned}
$$

As each $\Xi_{k}$ and $\Pi_{j}$ is a minimal $(P, R)$-module, we derive from Lemma 4.2 that the correspondence $\Xi_{k} \leftrightarrow \Pi_{k}$ is $1-1$.

Now $\Xi=\Xi^{\theta}+H^{\theta}$, where $H^{\theta}$ is the set of all $\xi$ annihilated by $e^{\theta}$. As $H^{\theta}=P\left(\xi_{1}\right.$ $\left.-e^{\theta} \xi_{1}, \xi_{2}-e^{\theta} \xi_{2}, \cdots\right)$,

$$
H^{\theta}=P \delta_{1}+P \delta_{2}+\cdots
$$

where $\delta_{i}=\xi_{j}-e^{\theta} \xi_{j}, j$ being a function of $i$. There exists a regular element $p$ of $\Delta$ defined by

$$
p \eta_{i k}=\zeta_{i k}, \quad i, k=1,2, \cdots ; \quad p \delta_{i}=\delta_{i}, \quad i=1,2, \ldots .
$$

For any $\alpha$ in $P, \bar{\alpha} \delta_{i}=\alpha \delta_{i}$ so that

$$
\bar{\alpha} p=p \bar{\alpha} .
$$

The inner automorphism $\tau$ defined by

$$
a^{r}=p^{-1} a p
$$

is a $\bar{P}$-automorphism.

From the definition of $p$, and as $s_{k}\left(\xi_{i}-e^{\theta} \xi_{i}\right)=u_{k}\left(\xi_{i}-e^{\theta} \xi_{i}\right)=0$ for all $k$ and $i$, it follows that

$$
\begin{aligned}
& u_{k} p \eta_{i k}=\xi_{i}=s_{k} \eta_{i k} ; \quad u_{k} p \eta_{i n}=0=s_{k} \eta_{i n}, \quad n \neq k ; i, k, n=1,2, \cdots, \\
& u_{k} p \delta_{i}=0=s_{k} \delta_{i}, \quad i, k=1,2, \cdots \text {. }
\end{aligned}
$$

Therefore

$$
s_{\dot{k}}=u_{k} p, \quad k=1,2, \cdots
$$

Similarly, as

$$
v_{k} \xi_{i}=\zeta_{i k}=p t_{k} \xi_{i}, \quad i, k=1,2, \cdots
$$

it follows that

$$
v_{k}=p t_{k}
$$

The above equations yield

$$
a^{\phi_{k}}=p^{-1} v_{k} a u_{k} p=a^{\theta_{k} \tau}, \quad k=1,2, \cdots .
$$

Thus $\phi_{k}=\theta_{k} \tau$ and the theorem follows.

6. Anti-endomorphisms of $\Delta$. In the case of a finite total matrix algebra over a division ring, the product of an anti-automorphism of the division ring with the transformation that takes every. element of the algebra into 
its transpose is an anti-automorphism of the algebra. Moreover, every antiautomorphism of the algebra can be expressed as the product of such an antiautomorphism with an inner automorphism $\left({ }^{\circ}\right)$.

If $a=\sum \bar{\alpha}_{r s} e_{r s}$ is an element of $\Delta$, it is not true in general that the transpose of $a, a^{T}=\sum \bar{\alpha}_{r e} e_{s r}$, is alșo an element of $\Delta$. Thus any anti-endomorphisms of $\Delta$ must be of a different type than those of a finite matrix algebra. Actually it will be shown that $\Delta$ has no nonzero anti-endomorphisms.

Let $\theta$ denote an anti-endomorphism of $\Delta$. Consider any infinite partition $\pi_{1}, \pi_{2}, \cdots$ of the set of positive integers into mutually exclusive subsets such that all $\pi_{i}$ contain an infinite number of elements. Define the elements $e_{i}$ of $\Delta$ by

$$
e_{i}=\sum_{j \in \pi} e_{j i}, \quad i=1,2, \cdots .
$$

In the proof of Theorem 3.3, the only products of elements of $\Delta$ used were commutative. The conclusions of the theorem are valid therefore for the anti-endomorphism $\theta$. Thus the set $e_{1}^{\theta}, e_{2}^{\theta}, \cdots$ is algebraically summable.

Denote the integers of $\pi_{k}$ by $n_{k 1}, n_{k 2}, \cdots$, and let

$$
r=\sum_{i, j=1}^{\infty} e_{j n_{i j}}, \quad s_{k}=\sum_{j=1}^{\infty} e_{n_{k j} j}, \quad k=1,2, \ldots .
$$

As $n_{i j} \neq n_{\imath k}$ for $j \neq k k$, both $r$ and $s$ are in $\Delta$. A simple multiplication yields

$$
r e_{k} s_{k}=e, \quad k=1,2, \cdots,
$$

and therefore

$$
s_{k}^{0} e_{k}^{\theta} \theta^{0}=e^{\theta}, \quad k=1,2, \ldots .
$$

Now in view of Theorem 2.4 , the set $s_{1}^{\theta} e_{1}^{\theta} r^{\theta}, s_{2}^{\theta} e_{2}^{\theta} r^{\theta}, \cdots$, that is, the set $e^{\theta}, e^{\theta}, \cdots$, is algebraically summable. However, the countably infinite set $e^{\theta}, e^{\theta}, \cdots$ is algebraically summable if and only if $e^{\theta}=0$. The anti-endomorphism $\theta$ must be the zero endomorphism, and we have proved the following theorem.

THEOREM 6.1. The ring $\Delta$ has no nonzero anti-endomorphisms.

(') N. Jacobson, The theory of rings, Mathematical Surveys vol. 2, New York, 1943, p. 23.

Mount Holyoke College,

SOUTh Hadley, Mass.

Purdue University,

LAFAYETTE, IND. 